\title{
The magnetic-field-induced transition from an expanding plasma to a double layer containing expanding plasma
}

\author{
C. Charles ${ }^{\text {a) }}$ and R. W. Boswell \\ Space Plasma, Power and Propulsion Group, Research School of Physical Sciences and Engineering, \\ The Australian National University, Australian Capital Territory 0200, Australia
}

(Received 20 September 2007; accepted 29 October 2007; published online 16 November 2007)

\begin{abstract}
The magnetic-field-induced transition from a simple expansion to a double layer is experimentally investigated in an argon low pressure radio frequency helicon source plasma. When the magnetic field is increased from 30 to $140 \mathrm{G}$ in the plasma source, an abrupt increase in the plasma density and upstream potential is measured at $50 \mathrm{G}$. In the downstream plasma, the plasma density and potential show a small decrease with increasing magnetic field and no abrupt change. When the upstream jump is measured, simultaneous measurements in the downstream plasma show an ion beam characteristic of a double layer near the source exit. (C) 2007 American Institute of Physics.
\end{abstract}

[DOI: $10.1063 / 1.2814877$ ]

Particle acceleration in magnetized expanding plasmas has been widely studied both in space and in laboratory plasmas. In space, ion heating and acceleration in the diverging magnetic field of coronal funnels at the surface of the sun has been measured by spectroscopy ${ }^{1}$ and ion and electron beams accelerated by magnetic-field-aligned electric fields called electric double layers (DLs) have been measured in the auroral cavity using electric probes on satellites. ${ }^{2}$ In the laboratory, ion acceleration downstream of low pressure high density magnetized plasma sources has been measured using energy analyzers and laser induced fluorescence without ${ }^{3}$ and with $^{4,5}$ an applied dc magnetic field in the source. The application of these phenomena to electric propulsion has been recently proposed. ${ }^{6}$ Except for strongly electronegative plasmas, ${ }^{7}$ double layers in low pressure, radio frequency (rf) generated expanding plasmas have been associated with the presence of a diverging magnetic field. ${ }^{6,8-10}$ However, the role of the magnetic field remains unclear and its detailed investigation is of importance for the further development of DL models ${ }^{11}$ and of plasma detachment studies. ${ }^{12}$

The previously described ${ }^{13}$ Chi-Kung experimental setup is shown in Fig. 1. The system is pumped down to a base pressure of $\sim 2 \times 10^{-6}$ Torr using a turbomolecular/rotary pumping system. The source consists of a Pyrex tube surrounded by a double-saddle field rf antenna extending between $z=3 \mathrm{~cm}$ and $z=21 \mathrm{~cm}$ ( $z$ is the reactor's axis and $z=30 \mathrm{~cm}$ is the source/chamber interface) and fed from a rf matching network/generator system operating at $13.56 \mathrm{MHz}$. Surrounding the tube and antenna, two solenoids centered at $z=3 \mathrm{~cm}$ (740 turns) and $z=21 \mathrm{~cm}$ (700 turns), respectively, are used to create a divergent magnetic field near the source exit; in this study the solenoids are fed by equal currents and this "solenoid current" is the parameter used for triggering and diagnosing the DL formation.

Three electric probes inserted via ports on the chamber back plate are used to characterize the plasma. A rfcompensated 3-mm-long 0.25-mm-diam cylindrical Langmuir probe $(\mathrm{CP})$ previously described ${ }^{14,15}$ is placed perpendicularly to the axis at $z=17 \mathrm{~cm}$, i.e., about $8 \mathrm{~cm}$ upstream of the DL to measure the plasma density, plasma potential, and

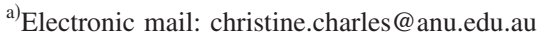

electron temperature. A non-rf compensated 2-mm-diam planar Langmuir probe (LP) is used to measure the plasma density along the $z$ axis (probe tip perpendicular to the axis) and a grounded retarding field energy analyzer (RFEA) placed at $z=37.5 \mathrm{~cm}$ with the collection aperture facing the source measures the ion energy distribution function (IEDF) downstream of the DL, using a deconvolution method previously described. ${ }^{13}$ For the DL case, a $0.63 \mathrm{~cm}$ in diameter optical periscope (somewhat smaller than that described in Ref. 16) looking radially with an acceptance angle of $\pm 5^{\circ}$, which can move freely inside a 1-cm-diam 70-cm-long Pyrex tube, is inserted along the axis via the chamber end plate. The optical emission signal of the $811.5 \mathrm{~nm}$ argon I line detected with a monochromator/photomultiplier (integrated along a radius) is measured along the axis and compared to the LP results.

Shown in Fig. 2 are axial measurements of the plasma density near the source center at $z=17 \mathrm{~cm}$ and at the source/ chamber interface $(z=30 \mathrm{~cm})$ for increasing solenoid current with a rf power of $400 \mathrm{~W}$ and a pressure of 0.3 mTorr. The density is derived from the planar LP ion saturation current (probe biased at $-54 \mathrm{~V}$ ) using an electron temperature of $8 \mathrm{eV}$ previously measured in the source for a pressure of 0.3 mTorr. ${ }^{6,14,15}$ The density at $z=17 \mathrm{~cm}$ is also measured using the compensated probe, also in ion saturation mode (filled triangles in Fig. 2). Below solenoid currents of 2 A, $n(17)$ and $n(30)$ are very similar and both are close to

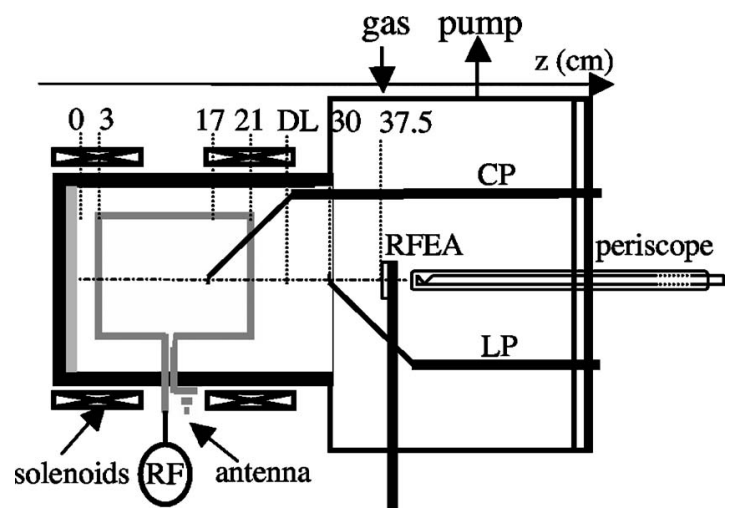

FIG. 1. Schematic of the Chi-Kung plasma source $(31 \mathrm{~cm}$ by $13.7 \mathrm{~cm})$ and grounded chamber $(31.8 \mathrm{~cm}$ by $29.4 \mathrm{~cm})$ showing probes. 


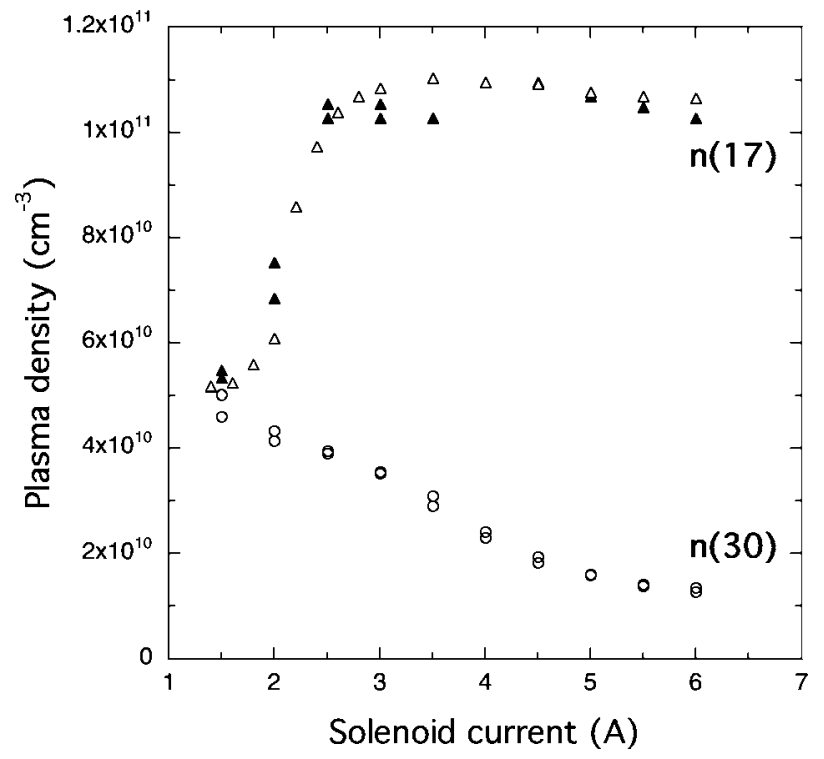

FIG. 2. Plasma density vs solenoid current measured at $z=17 \mathrm{~cm}$ with the LP (open triangles) and the CP (solid triangles) and at $z=30 \mathrm{~cm}$ with the LP (open circles); the operating conditions are an argon pressure of 0.3 mTorr and a rf power of $400 \mathrm{~W}$.

$5 \times 10^{10} \mathrm{~cm}^{-3}$. For solenoid currents between 2 and $2.5 \mathrm{~A}, \mathrm{a}$ dramatic increase in $n(17)$ by a factor of about 2 to a value of $\sim 10^{11} \mathrm{~cm}^{-3}$ is measured followed by no further increase when the current is increased from 3 to $6 \mathrm{~A}$. In contrast to this behavior, the variation in $n(30)$ is a gradual decrease by a factor of about 5 from $5 \times 10^{10} \mathrm{~cm}^{-3}$ to $\sim 10^{10} \mathrm{~cm}^{-3}$ when the current is increased from 1.5 to $6 \mathrm{~A}$. For a current of $6 \mathrm{~A}$, the $n(17) / n(30)$ ratio is about 7.

Normalized IEDFs measured with the RFEA at $37.5 \mathrm{~cm}$ for increasing solenoid current are shown in Fig. 3 for a pressure of $0.3 \mathrm{mT}$ Torr and a somewhat higher power of $550 \mathrm{~W}$. Below $2 \mathrm{~A}$, a single peaked IEDF at $30 \mathrm{~V}$ is obtained, which corresponds to the local plasma potential at the RFEA on-axis position. Since the RFEA is grounded, the peak at $30 \mathrm{~V}$ corresponds to ions falling through the sheath at the entrance of the analyzer and reflects nearly zero energy of these ions at $z=37.5 \mathrm{~cm}$. Above $2.4 \mathrm{~A}$, a second peak at higher energy $(35-50 \mathrm{~V})$ is measured in addition to the low energy peak $(25-30 \mathrm{~V})$. This high energy peak is a well defined ion beam for solenoid currents above $3.5 \mathrm{~A}$. Both the ion beam energy and current increase with increasing solenoid current. The ion beam is the marker for the formation of a double layer near the exit of the source and corresponds to acceleration by the DL potential drop of the ions formed upstream of the DL, i.e., near the source center. 6,15

The plasma potential $V_{p}$ obtained at $z=17 \mathrm{~cm}$ using the maximum in the first and the zero crossing in the second derivative of the rf-compensated Langmuir probe currentvoltage characteristic is shown by circles and crosses in Fig. 3 , respectively. For all solenoid currents, the upper energy threshold ( 0.1 in the color scale) in the IEDF tracks the plasma potential: above the transition at $2.5 \mathrm{~A}$, the upper energy threshold corresponds to the fastest ions within the ion beam, about $10 \mathrm{~V}$ higher than the flux peak in the beam energy. The mean (peak flux) beam energy corresponds to the plasma potential at the upstream edge of the double layer $(z \sim 25 \mathrm{~cm})$ : higher energy ions within the beam come further upstream (typically for $z$ values from 17 to $25 \mathrm{~cm}$ ) but Downloaded 09 Apr 2008 to 130.56.65.24. Redistribution subject

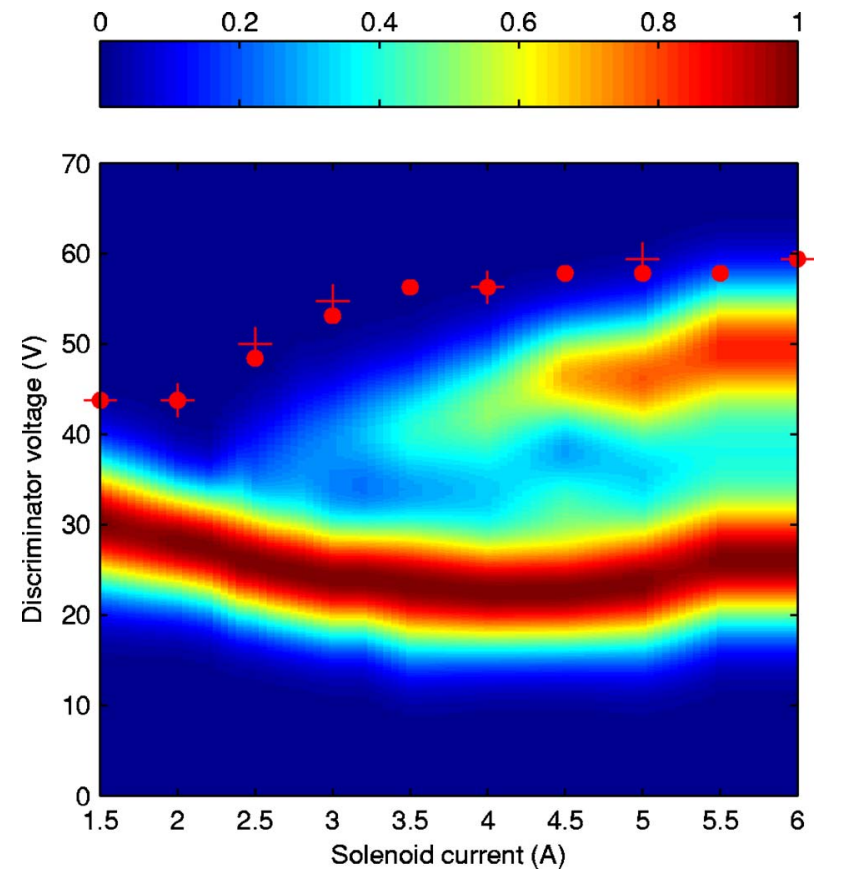

FIG. 3. (Color online) Normalized IEDF (color bar) vs solenoid current measured with the RFEA positioned at $z=37.5 \mathrm{~cm}$ and plasma potential vs solenoid current obtained with the CP positioned at $z=17 \mathrm{~cm}$ from the first derivative (filled red circles) and from the zero crossing in the second derivative (red crosses); same operating conditions as in Fig. 2 except for a higher rf power of $550 \mathrm{~W}$ for the RFEA measurements. The change from 400 to $550 \mathrm{~W}$ only induces an increase of the signal on the RFEA collector.

their number is less due to ion-neutral collisions between where they were created and where they were detected. At a pressure of 0.3 mTorr for argon, the mean free path is about $8 \mathrm{~cm}$.

Figure 3 shows that the difference in plasma potential between $z=17 \mathrm{~cm}$ and $z=37.5 \mathrm{~cm}$ is greatest for $6 \mathrm{~A}$ and is likely related to the corresponding maximum in density difference between $z=17 \mathrm{~cm}$ and $z=30 \mathrm{~cm}$ measured for $6 \mathrm{~A}$ (Fig. 2). To further investigate the effect of the rf antenna geometry and magnetic field structure, the density is measured along the source axis for magnetic field strengths before $(2 \mathrm{~A})$ and after ( 4 and $6 \mathrm{~A})$ the transition and the results are shown in Fig. 4, respectively. The $B_{z}$ component of the magnetic field is plotted in Fig. 4 for the $6 \mathrm{~A}$ case (the shape is conserved for the 2 and $4 \mathrm{~A}$ cases). For a current of $2 \mathrm{~A}$, i.e., before the transition to a DL containing plasma, the density is maximum at about $z=20 \mathrm{~cm}$ and above the transition (4 and $6 \mathrm{~A}$ cases), the density is maximum in the region corresponding to the middle of the rf antenna at $z=12 \mathrm{~cm}$, a region which also corresponds to the magnetic field minimum between the two maxima at $z=3 \mathrm{~cm}$ and $z=21 \mathrm{~cm}$.

The pretransition plasma appears little affected by the presence or position of the magnetic field or the rf antenna and the plasma densities in the source and diffusion chamber are similar. After the transition, the source plasma density dramatically increases while the downstream plasma gently decreases in density. For such an increase in density to occur without a change in $\mathrm{rf}$ power, the upstream loss rate must have decreased by a factor corresponding to the increase in density. As the transition appears to be provoked by the increasing magnetic field, it is likely that loss of plasma to the source walls has somehow been decreased. The present results suggest that there are two distinct regions, a region of AIP license or copyright; see http://apl.aip.org/apl/copyright.jsp 


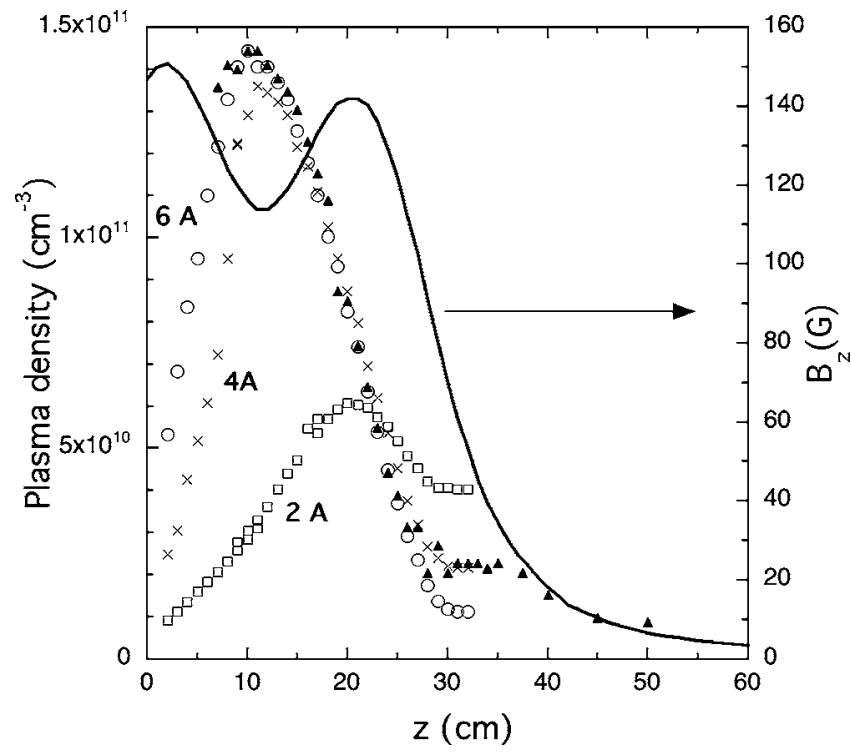

FIG. 4. Plasma density profiles on axis obtained with the LP for 2 A (open squares), $4 \mathrm{~A}$ (crosses), and $6 \mathrm{~A}$ (open circles) for a rf power of $400 \mathrm{~W}$; optical emission signal of the $811 \mathrm{~nm}$ argon I line measured with the periscope for $6 \mathrm{~A}$ (filled triangles) for a rf power of $250 \mathrm{~W}$ and normalized for a rf power of $400 \mathrm{~W}$; magnetic field profile $B_{z}$ (solid line) measured with the Hall probe for $6 \mathrm{~A}$; the operating pressure is $0.3 \mathrm{mT}$ Torr.

ionization and a (probably narrow) region of acceleration. This has not been considered in associated theoretical studies. ${ }^{11,17}$

The cross field diffusion for these low magnetized plasmas is generally dominated by the ion drifts. At $z=12 \mathrm{~cm}$, between the two solenoids, the measured $B_{z}$ 's are 40,77 , and $114 \mathrm{G}$ for the 2, 4, and $6 \mathrm{~A}$ cases, respectively, yielding a Larmour radius for the ions of 7.2, 3.7, and $2.5 \mathrm{~cm}$, respectively. A previously measured ion temperature of $0.2 \mathrm{eV}$ in the source is used for the ion Larmour radius calculation. ${ }^{18}$ The start of the transition in the upstream density shown in Fig. 2 corresponds to a solenoid current of $2 \mathrm{~A}$, i.e., to an ion Larmour radius close to $7 \mathrm{~cm}$ in the $\mathrm{rf}$ antenna region, a value similar to the inner source tube radius of $6.85 \mathrm{~cm}$. Above the transition, the ion Larmour radius becomes smaller than the inner tube radius, reducing the radial loss rate to the source walls and resulting in an increase in central density and, more importantly, for the formation of the double layer, an increase of the density gradient from the source center to the open end of the source. Before the transition, the density $n(17)$ is lower, the maximum density is located at about $z=20 \mathrm{~cm}$, the density gradient from the maximum in density to the exit of the source is less and no ion beam is observed.

Optical measurements with the periscope are carried out for the standard DL parameters $(250 \mathrm{~W}, 0.3 \mathrm{mTorr}$, and $6 \mathrm{~A})$ previously reported ${ }^{13}$ using the $811 \mathrm{~nm}$ argon line and the results normalized at $z=12 \mathrm{~cm}$ for the $400 \mathrm{~W}$ case and plotted as solid triangles in Fig. 4. The results are very similar to the density measurements made by the Langmuir probe which is to be expected from the simple model assuming direct excitation from the neutral ground state: $\left(I_{\mathrm{ArI}}\right) \sim n_{e}$ $\times n_{0} \times f\left(T_{e}\right)$, where $\left(I_{\mathrm{ArI}}\right)$ is the intensity of the $811 \mathrm{~nm}$ argon line, $n_{e}$ is the plasma density, $n_{0}$ is the neutral density, and $f\left(T_{e}\right)$ is a complicated function of the electron energy distribution function involving atomic transition processes. Interestingly, the optical results start diverging from the Langmuir probe results at about $z=25 \mathrm{~cm}$ (the DL position) and the flat response of the periscope from $z \sim 28 \mathrm{~cm}$ to $z \sim 38 \mathrm{~cm}$ corresponds to the region of maximum beam intensity. It has been previously shown that at $z=45 \mathrm{~cm}$, the beam intensity is low as a result of ion-neutral charge exchange collisions. $^{13}$

In summary, this experimental study demonstrates that a sufficient axial magnetic field will trigger the formation of a double layer in a low pressure expanding plasma. This results in an increased density in the plasma source and, hence, an increased density gradient at the exit of the plasma source which leads to the formation of a double layer and ion beam. For the present source geometry this can be achieved by applying a minimum magnetic field of about $50 \mathrm{G}$ in the source. Above the magnetic-field-induced transition, the plasma density and the plasma potential are maximum in the center of the source where both the rf antenna and maximum magnetic field regions are situated. The maximum energy of the ion beam formed by acceleration in the double layer correlates well with the large plasma potential measured near the source center. A possible reason for this behavior is the magnetic field which reduces the Larmour radius of the radially diffusing ions until, at the transition, the Larmour radius is equal to the tube diameter and any further increase in magnetic field will result in a reduced Larmour radius and reduced cross field diffusion. The resulting increase in the source density leads to large density and potential gradients along the axis which are conditions propitious to the formation of a double layer.

${ }^{1}$ R. Esser, O. Lie-Svendsen, A. M. Janse, and M. A. Killie, Astrophys. J. 629, L61 (2005).

${ }^{2}$ R. E. Ergun, L. Andersson, D. S. Main, Y. J. Su, C. W. Carlson, J. P. McFadden, and F. S. Mozer, Phys. Plasmas 9, 3685 (2002).

${ }^{3}$ C. Charles, R. W. Boswell, and R. K. Porteous, J. Vac. Sci. Technol. A 10, 398 (1992).

${ }^{4}$ R. A. Gottscho, T. Nakano, N. Sadeghi, D. J. Trevor, and R. W. Boswell, Proc. SPIE 1594, 376 (1991).

${ }^{5}$ C. Charles, Appl. Phys. Lett. 84, 332 (2004).

${ }^{6}$ C. Charles and R. W. Boswell, Appl. Phys. Lett. 82, 1356 (2003).

${ }^{7}$ N. Plihon, C. S. Corr, and P. Chabert, Appl. Phys. Lett. 86, 091501 (2005).

${ }^{8}$ S. A. Cohen, N. S. Siefert, S. Stange, R. F. Boivin, E. E. Scime, and F. M. Levinton, Phys. Plasmas 10, 2593 (2003).

${ }^{9}$ X. Sun, A. M. Keesee, C. Biloiu, E. E. Scime, A. Meige, C. Charles, and R. Boswell, Phys. Rev. Lett. 95, 025004 (2005).

${ }^{10}$ O. Sutherland, C. Charles, N. Plihon, and R. W. Boswell, Phys. Rev. Lett. 95, 205002 (2005).

${ }^{11}$ M. A. Lieberman, C. Charles, and R. W. Boswell, J. Phys. D 39, 3294 (2006).

${ }^{12}$ E. B. Hooper, J. Propul. Power 9, 757 (1993).

${ }^{13}$ C. Charles and R. W. Boswell, Phys. Plasmas 11, 1706 (2004).

${ }^{14}$ K. Takahashi, C. Charles, R. W. Boswell, T. Kaneko, and K. Hatakeyama, Phys. Plasmas 14, 1 (2007).

${ }^{15}$ C. Charles, Plasma Sources Sci. Technol. 16, R1 (2007).

${ }^{16}$ J. Scharer, A. Degeling, G. Borg, and R. W. Boswell, Phys. Plasmas 9, 3734 (2002).

${ }^{17}$ A. Fruchtman, Phys. Rev. Lett. 96, 065002 (2006).

${ }^{18}$ A. M. Keese, E. E. Scime, C. Charles, A. Meige, and R. W. Boswell, Phys. Plasmas 12, 093502 (2005). 